Bull. Mater. Sci., Vol. 8, No. 1, February 1986, pp. 39-48. (C) Printed in India.

\title{
Magnetic and Mössbauer studies on ductile Fe-Cr-Co permanent magnet alloys
}

\author{
V CHANDRASEKARAN, CH SREERAMA MURTHY, \\ P SUBRAHMANIAM and G VENKATESWARLU \\ Defence Metallurgical Research Laboratory, P.O. Kanchanbagh, Hyderabad 500258, India
}

MS received 28 October 1985

\begin{abstract}
Iron-chromium-cobalt alloys possess attractive magnetic properties combined with good formability and hence are identified as technologically important magnetic materials. Alloys with compositions Fe-28.9 Cr-15.6 Co and Fe-28.4 Cr-20-1 Co (weight percent) have been studied. Heat-treatment parameters during thermomagnetic treatment viz temperature, time and external magnetic field were optimized with reference to magnetic properties. The fully treated anisotropic alloys develop remanence $=11.5-12.0$ kilo Gauss, coercivity $=600-650$ Oersted and energy product $=4-4-5$ million Gauss Oersted. Electron microscopic and Mössbauer spectroscopic techniques were used to identify the original and transformed phases. During the various stages of the development of the alloy, the changes in mechanical hardness were correlated with magnetic hardness.
\end{abstract}

Keywords. Magnetic properties; heat treatments; phase identification; development of ductile permanent magnet; Mössbauer studies; iron-chromium-cobalt alloys.

\section{Introduction}

Iron-chromium-cobalt magnetic alloys, first reported by Kaneko et al (1971) combine excellent permanent magnetic properties with good ductility. The magnetic properties such as remanence $\left(B_{r}\right)$, coercivity $\left(H_{c}\right)$ and energy product $(\mathrm{BH})_{\max }$ obtained in these alloys are comparable (Chin 1980) to those of Alnico $\mathrm{V}$, a conventional magnetic material widely used for many applications. The Fe-Cr-Co alloy, completely free from nickel, needs less cobalt than the brittle Alnico $\mathrm{V}$ and hence has been identified as technologically important magnetic material (Chin 1978). Due to these superior features, considerable effort has been made for the development and better understanding of these alloys (Belli et al 1978; Chin 1978; 1980; Chin et al 1978, $1983 \mathrm{a}, \mathrm{b}$; Cremer and Pfeiffer 1975; Gin and Gayle 1980; Green et al 1980; Homma 1977; Houghton et al 1978; Kaneko et al 1972, 1975 a, b, 1976, 1977; Livingston 1981; Mahajan et al 1978; Minowa et al 1980; Okada et al 1978; Sherwood et al 1979; Tin 1979; Tin et al 1980 a, b; Wang Run et al 1984; Zijlstra 1978). Studies carried out in the course of development of two alloys, namely $\mathrm{Fe}-28 \cdot 9 \mathrm{Cr}-15 \cdot 6 \mathrm{Co}$ and $\mathrm{Fe}-28 \cdot 4 \mathrm{Cr}-20 \cdot 1 \mathrm{Co}$, are presented and discussed in this paper.

\section{Experimental}

Armco iron, electrolytic cobalt and chromium in appropriate quantities were melted in magnesia crucibles under a dynamic vacuum of $10^{-3}$ torr in a $15 \mathrm{~kW}, 8000 \mathrm{~Hz}$. Stokes vacuum induction furnace. The alloy in each case was poured at $1650^{\circ} \mathrm{C}$ into preheated 


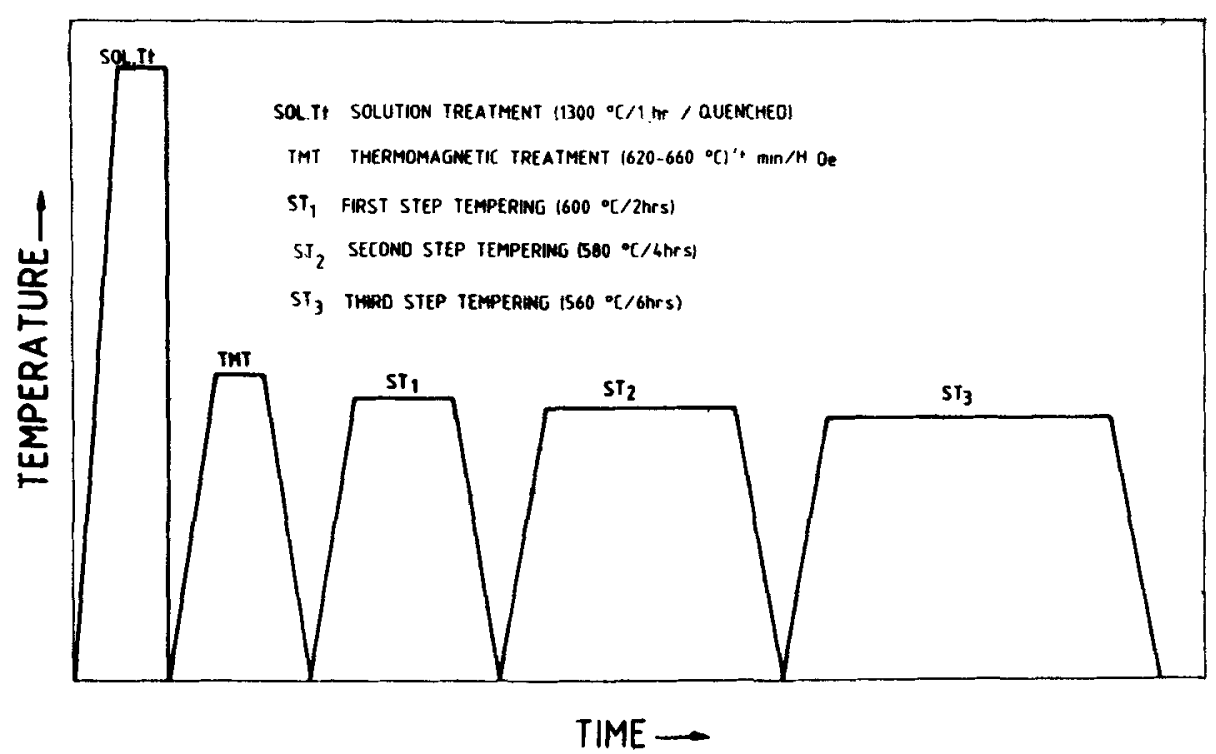

Figure 1. Heat treatment schedule for Fe-Cr-Co magnetic alloys.

$25 \mathrm{~mm}$ round cast iron moulds. The ingots were processed by hot forging and hot rolling at $1250^{\circ} \mathrm{C}$ to $12.7 \mathrm{~mm}$ dia rods and test samples of the size $10 \mathrm{~mm}$ dia $\times 15 \mathrm{~mm}$ long were prepared. The chemical composition of the two alloys by wt $\%$ was as follows: Fe-28-9Cr-15.6Co (alloy-1) and $\mathrm{Fe}-28 \cdot 4 \mathrm{Cr}-20 \cdot 1 \mathrm{Co}$ (alloy-2). The heat-treatment procedure adopted in the present study is schematically given in figure 1 . The samples were solution-treated at $1300^{\circ} \mathrm{C}$ for one hour and quenched in brine water to retain the high temperature single phase (bcc phase) at room temperature. Optical metallography and $x$-ray diffraction techniques were used to confirm the presence of this single phase. To carry out the critical thermomagnetic treatment (TMT), an isothermal treatment in a magnetic field, a small Kanthal-wire-wound furnace was fabricated and housed between the poles of a heavy duty electromagnet capable of generating a uniform magnetic field of 3750 Oersted. The important parameters controlled during TMT were: (i) the temperature, (ii) soaking time and (iii) the strength of the external magnetic field.

Experiments were carried out to optimize these parameters keeping two of the variables constant at a time and varying the third. The samples were subsequently steptempered at $600^{\circ} \mathrm{C} / 2 \mathrm{hr}, 580^{\circ} \mathrm{C} / 4 \mathrm{hr}$ and $560^{\circ} \mathrm{C} / 6 \mathrm{hr}$ as given in figure 1 . After every stage of the heat-treatment, the magnetic properties of the samples were measured using a double yoke ferrotester and the hardness of the alloys was also measured. Electron microscope was used to note the morphology of the phases formed during the treatment. For Mössbauer studies, solution-treated samples of alloy-1, electrochemically thinned to $50 \mu \mathrm{m}$ thickness, were encapsulated in evacuated quartz tube and subjected to all the treatments as shown in figure 1. However, since the samples were thin foils, the duration of the treatments was suitably reduced to avoid overaging and the following schedule was used: $635^{\circ} \mathrm{C} / 20^{\prime} / 2000 \mathrm{Oe}, 600^{\circ} \mathrm{C} / 1 \mathrm{hr}, 580^{\circ} \mathrm{C} / 2 \mathrm{hr}$, $560^{\circ} \mathrm{C} / 3 \mathrm{hr}$ was followed. The Mössbauer transmission spectrum after each stage of treatment was recorded at room temperature using ECIL Mössbauer spectrometer 
(MBS 35) with 10 milli Curie strength ${ }^{57}$ Co radioactive source in palladium matrix in the constant acceleration mode.

\section{Results and discussion}

The magnetic properties $\left(B_{r}, H_{c}\right.$ and $\left.[\mathrm{BH}]_{\max }\right)$ of the two alloys after complete step tempering treatments have been plotted in figures $2 \mathrm{a}, \mathrm{b}, \mathrm{c}$ for the experimental conditions indicated therein. It can be seen from figure $2 a$ that the magnetic properties, especially coercivity and energy product are sensitive to the changes in the temperature of TMT and the variation is parabolic. In alloy $1, B_{r}$ remains constant throughout the treatment while in alloy 2 a slight decrease in $B_{r}$ at high temperature has been observed. The maximum $H_{c}$ is obtained at the spinodal decomposition (Homma 1977) temperature $635^{\circ} \mathrm{C}$ and $645^{\circ} \mathrm{C}$ for alloys 1 and 2 respectively. The observed increase in the decomposition temperature for alloy 2 is due to the increase in cobalt content (Chin 1978).

The spinodal decomposition in Fe-Cr-Co alloy, at temperatures below $700^{\circ} \mathrm{C}$, leading to the high temperature single phase $\alpha$ decomposing into two isomorphous phases $\alpha_{1}$ (rich in Fe and $\mathrm{Co}$ ) and $\alpha_{2}$ (rich in $\mathrm{Cr}$ ) has been investigated by earlier workers (Homma 1977; Okada et al 1978; Wang Run et al 1984). Due to the difference in their chemical composition, the two phases possess different values of saturation magnetisation and curie temperature. During the step-tempering treatment, diffusion of $\mathrm{Fe}$ and

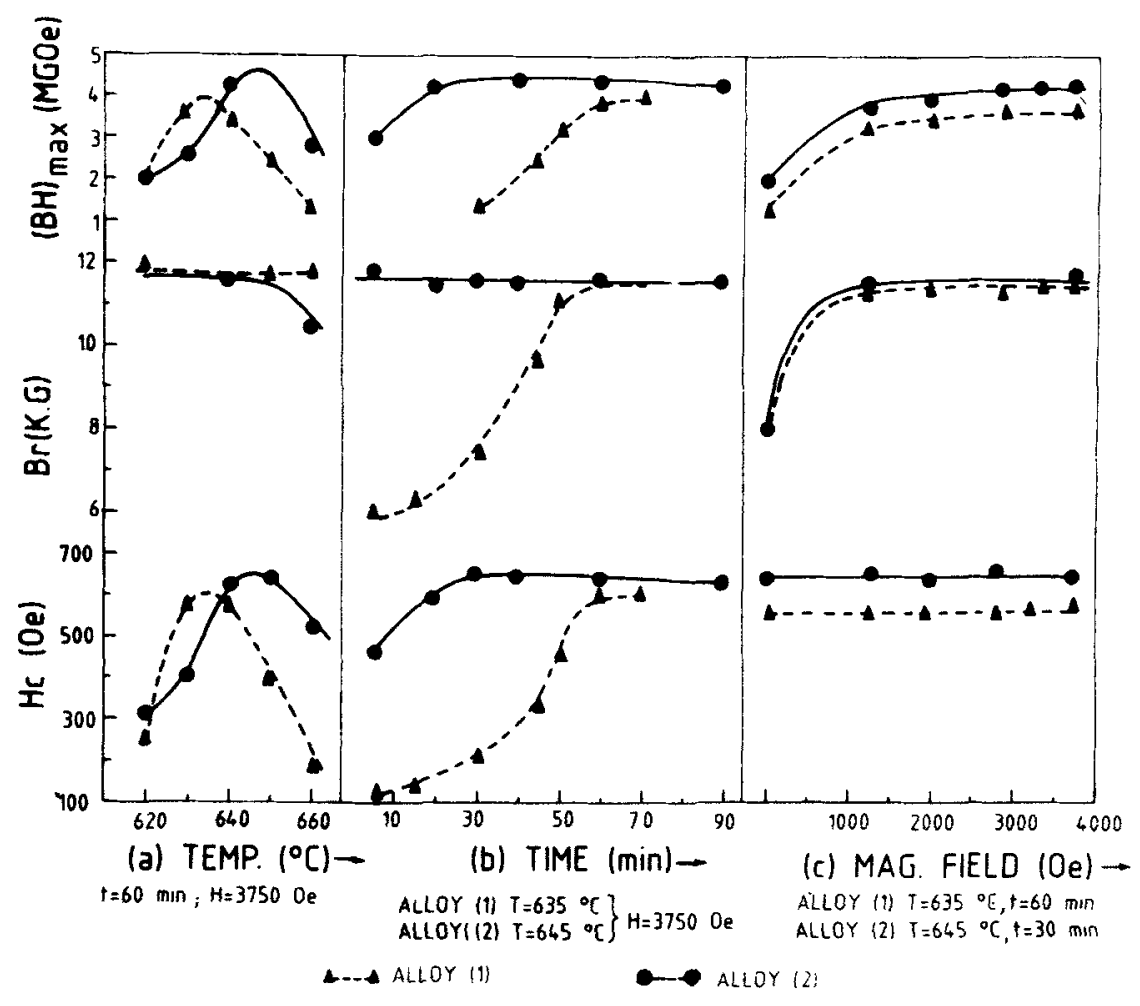

Figure 2. Magnetic properties of fully treated Fe-Cr-Co alloys vs TMT parameters. 
Co from $\alpha_{1}$ to $\alpha_{2}$ occurs (Okada et al 1978) thus making $\alpha_{1}$ strongly ferromagnetic and $\alpha_{2}$ paramagnetic. The presence of magnetic and non-magnetic phases and the complex domain wall interactions account for the high coercivity in these alloys (Mahajan et al 1978; Livingston 1981). For a group of oriented ellipsoidal single domain particles in a less magnetic matrix, Stoner and Wohlfarth (1948) derived the following relationship for coercivity:

$$
H_{c}=p(1-p)\left(N_{2}-N_{1}\right)\left(I_{1}-I_{2}\right)^{2} / I_{s}
$$

where $N_{1}$ and $N_{2}$ are demagnetization factors along major and minor axes respectively; $I_{1}$ and $I_{2}$, saturation magnetization of the ellipsoid and the matrix respectively; $I_{s}$, the average saturation magnetization and $p$, the volume fraction of the particles.

The demagnetization factors explain the magnetic anisotropy whereas the volume fractions and the saturation magnetization values account for the mechanism of domain wall motion for coercivity. This theory has been widely accepted (Mason et al 1970) for Alnico alloys. Since Fe-Cr-Co alloys are akin to Alnicos in achieving high magnetic hardness, the present experimental observations on the coercivity could be understood on the basis of the Stoner and Wohlfarth theory. Due to the asymmetric shape of the miscibility gap in Fe-Cr-Co system (Kaneko et al 1977; Minowa et al 1980) the volume fractions of the $\alpha_{1}$ and $\alpha_{2}$ change with TMT temperature. It has been reported (Okada et al 1978) that during the step-tempering treatments, the morphology and volume fractions of the phases essentially remain unchanged. Hence the ultimate volume fractions of the phases which control the coercivity through Stoner and Wohlfarth relationship mainly depend on the temperature of TMT, thus explaining the parabolic variation of $H_{c}$ and $(\mathrm{BH})_{\max }$.

Figure $2 \mathrm{~b}$ shows that $H_{c}$ of the alloys increases with soaking time when treated at optimized temperatures. A minimum period of $60^{\prime}$ and $30^{\prime}$ are needed for the alloys 1 and 2 respectively to maximize coercivity by imparting magnetic anisotropy and completing the decomposition of $\alpha$ phase to the required level. The decreasing trend in $H_{c}$ due to prolonged treatment can be noted from the curve for alloy 2 . The need for more soaking time for the low cobalt alloy is due to slower kinetics at lower temperature. During the initial stages, this alloy exhibits lower values of remanence which may be due to the isotropic nature (Okada et al 1978) of products of the decomposition. The improvement in magnetic anisotropy and completion of the decomposition reaction which alter the demagnetization factors and saturation magnetization values of the phases increase the coercivity as expected from the Stoner and Wohlfarth relation.

Figure $2 \mathrm{c}$ shows the strong dependence of $B_{r}$ on the external magnetic field applied during the treatment. A significant increase from $8000 \mathrm{G}$ to $12000 \mathrm{G}$ in $B_{r}$ is observed due to the presence of the field. The purpose of the application of an external field during TMT is to align the phases and elongate them in its direction and this effect can be observed from the electron micrographs (carbon replica) for the alloy 1 presented in figures $3 \mathrm{a}, \mathrm{b}$. The coalescence of the spherical phases in the form of ellipsoids elongated in the direction of the field not only improves $B_{r}$ but also enhances magnetic anisotropy. A minimum field of $1500-2000 \mathrm{Oe}$ is essential to maximize $B_{r}$. Though from figure $2 \mathrm{c}$ no apparent effect of magnetic field on coercivity is seen, figure 4 (Curves 4 and 5) however shows that the magnetic field affects the shape of the demagnetization curves. The 'knee of the demagnetization curve' is absent in samples treated without the 


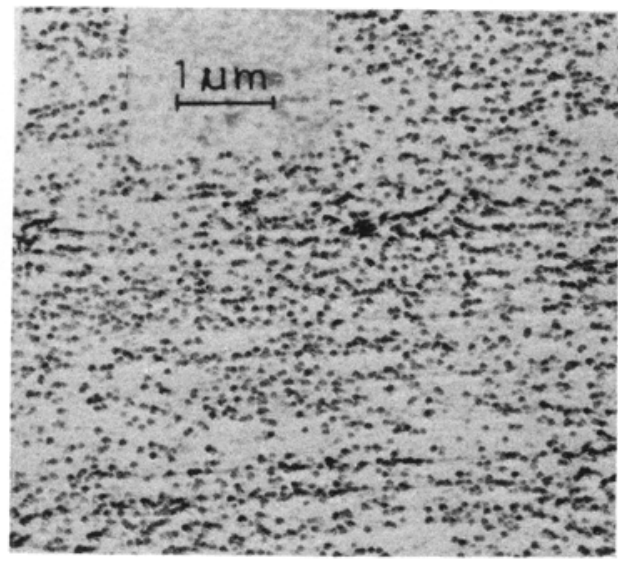

(a)

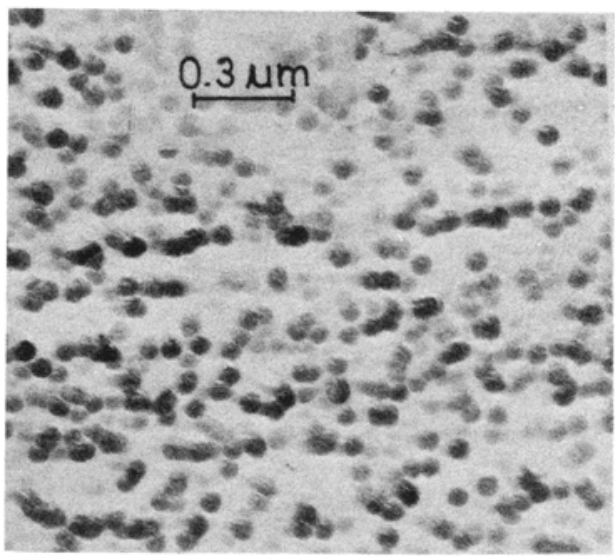

(b)

Figure 3. Carbon replica micrograph of alloy-1. (a) Spherical morphology of the decomposed phase aligned along the direction of the applied field. (b) Coalescence of the phases to ellipsoid shape.

magnetic field which indicates the absence of strong magnetic anisotropy in these samples.

The gradual improvement in coercivity with step tempering treatments is shown in figure 4. The observed increase in $H_{c}$ is associated with the increase in the difference in the saturation magnetization values of $\alpha_{1}$ and $\alpha_{2}$ phases upon step-tempering through diffusion-controlled composition variations in the phases. Thus thermomagnetic treatment plays the important role of fixing the volume fractions and magnetic anisotropy while the step-tempering treatments help in widening the difference in saturation magnetization values between the phases. Hence the Stoner-Wohlfarth relation for coercivity has been found to fittingly explain the experimental observations in achieving magnetic properties of $\mathrm{Fe}-\mathrm{Cr}-\mathrm{Co}$ alloys. The isotropic and anisotropic magnetic properties of the fully treated alloys are listed in table 1.

The close relationship between mechanical and magnetic hardness is established through figure 5 in which the values are plotted stagewise. In the solution-treated condition, the alloys are mechanically soft and machining operations are possible in 


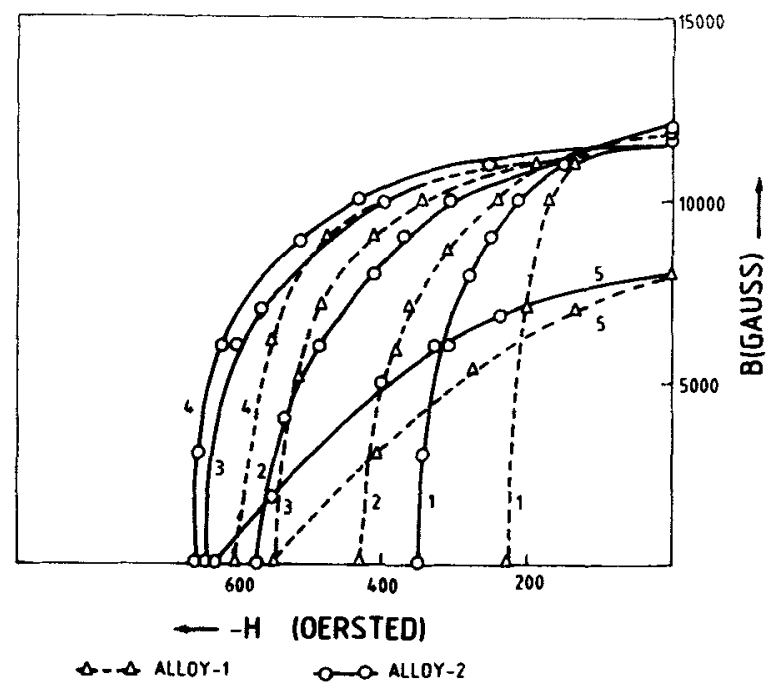

(1) AFTER TMT, (2) AFTER TMT+ST , (3) AFTER YMT+ST, $+S T_{2}$,

(4) AFTER TMT +ST, +ST • ST3, (5) FULLY TREATEO WITHOUT MAGNETIC

FIELD OURING TMT.

Figure 4. Second quadrant demagnetization curves for Fe-Cr-Co alloys. The gradual improvement in coercivity, shape of the curve upon heat treatment (curves 1 to 4) and the absence of 'knee of the demagnetization curve' in isotropic magnets (curve 5) can be observed.

Table 1. Permanent magnetic properties of Fe-Cr-Co alloys

\begin{tabular}{lccc}
\hline Alloy & $\begin{array}{c}\text { Remanence } \\
B_{r} \text { (gauss) }\end{array}$ & $\begin{array}{c}\text { Coercivity } \\
H_{c} \text { (Oersted) }\end{array}$ & $\begin{array}{c}\text { Energy product } \\
(\mathrm{BH})_{\max } \text { (MGOe) }\end{array}$ \\
\hline $\begin{array}{l}\text { Fe-28.9Cr-15.6Co } \\
\text { (a) Anisotropic }\end{array}$ & $11750 \pm 250$ & $600 \pm 25$ & \\
(b) Isotropic & $8250 \pm 250$ & $500 \pm 25$ & $1 \cdot 0 \pm 0.2$ \\
Fe-28-4Cr-20.1Co & & & \\
(a) Anisotropic & $11750 \pm 250$ & $650 \pm 25$ & $4 \cdot 5 \pm 0.2$ \\
(b) Isotropic & $8250 \pm 250$ & $625 \pm 25$ & $2 \cdot 0 \pm 0.2$ \\
\hline
\end{tabular}

this condition. As a result of the heat treatments, due to the decomposition of $\alpha$-phase and enrichment of chromium in $\alpha_{2}$ the values of mechanical as well as magnetic hardness increase simultaneously.

\subsection{Mössbauer studies}

In the $\mathrm{Fe}-\mathrm{Cr}$-Co system, due to the magnetic phases available after TMT and the constant changes in the magnetic nature of the phases during step-tempering treatments, Mössbauer spectroscopy has been effectively utilised (Houghton et al 1978; Chen Ming-Yan et al 1982) to understand the decomposition mechanisms.

In figure 6 are presented the Mössbauer spectra of alloy 1 along with the spectrum of $\alpha-\mathrm{Fe}$ for reference and to serve as a calibration standard for the estimation of hyperfine 


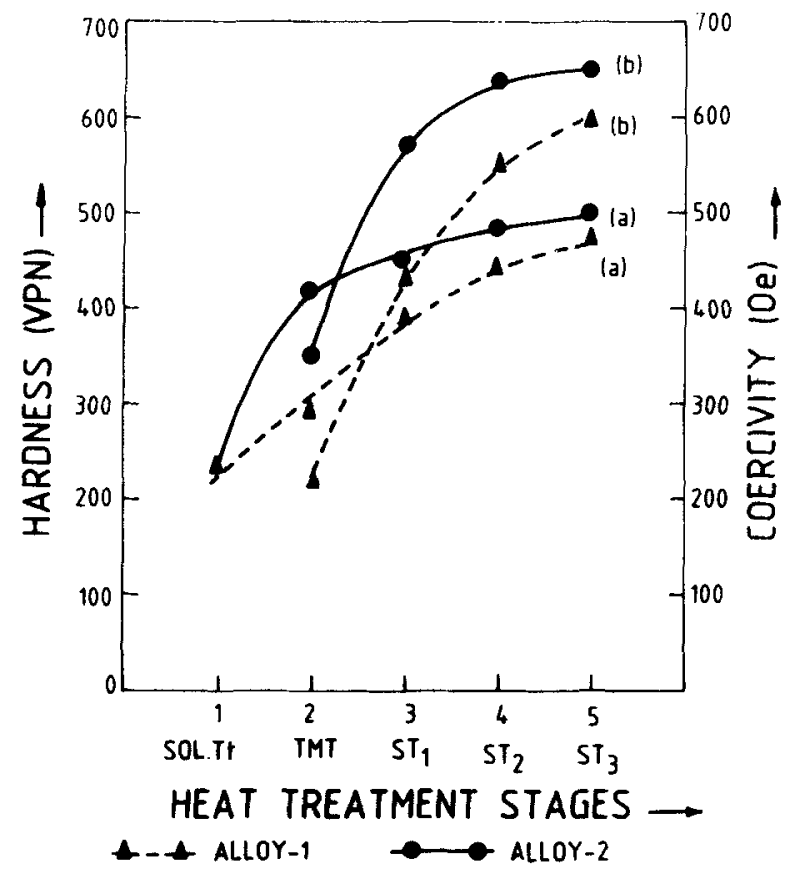

Figure 5. Improvement in mechanical (a) and magnetic (b) hardness in Fe-Cr-Co alloys upon heat treatment.

fields. The spectrum at 6-B of the solution-treated sample shows single phase structure $(\alpha)$ of the alloy. Broad distribution of hyperfine fields and the small isomer shift indicate randomized atomic distribution within the single phase. The hyperfine field has been found to be centred near about $250 \mathrm{KOe}$. From the spectrum at 6-C which pertains to TMT, there is evidence for two hyperfine fields of $355 \mathrm{KOe}$ and $202 \mathrm{KOe}$ corresponding to the two phases $\alpha_{1}$ and $\alpha_{2}$ respectively both being ferromagnetic with different magnitudes of intensity of magnetization. The corresponding two six-line spectra could not be resolved because of overlapping (Houghton et al 1978) of outer lines of smaller field with the second and fifth lines of larger field. The existence of $\alpha_{2}$ in paramagnetic state is indicated by the presence of a small peak at the centre of the spectrum. The spectra from 6-D to F correspond to those for step-tempering treatments. The gradual increase in the spectral area of the central paramagnetic peak and the narrowing of the other lines indicate that the volume fraction of paramagnetic $\alpha_{2}$ increases with steptempering. This is due to the progressive conversion of $\alpha_{2}$ from weakly ferromagnetic to paramagnetic state.

Under the present experimental conditions, (with thickness of sample being around $50 \mu \mathrm{m}$ ) it will be difficult to quantitatively correlate the spectral areas to the volume fractions of the phases. However, an attempt has been made to derive qualitative correlations. The relative area of central line of $\alpha_{2}$ to the total area of the spectrum has been found to be $0.03,0.06$ and 0.10 for the three step-tempering treatments at $600^{\circ}$, $580^{\circ}$ and $560^{\circ} \mathrm{C}$ respectively indicating the increase in the amount of paramagnetic $\alpha_{2}$. The observed increase in coercivity with these treatments (figure 4) can be explained on this basis. 


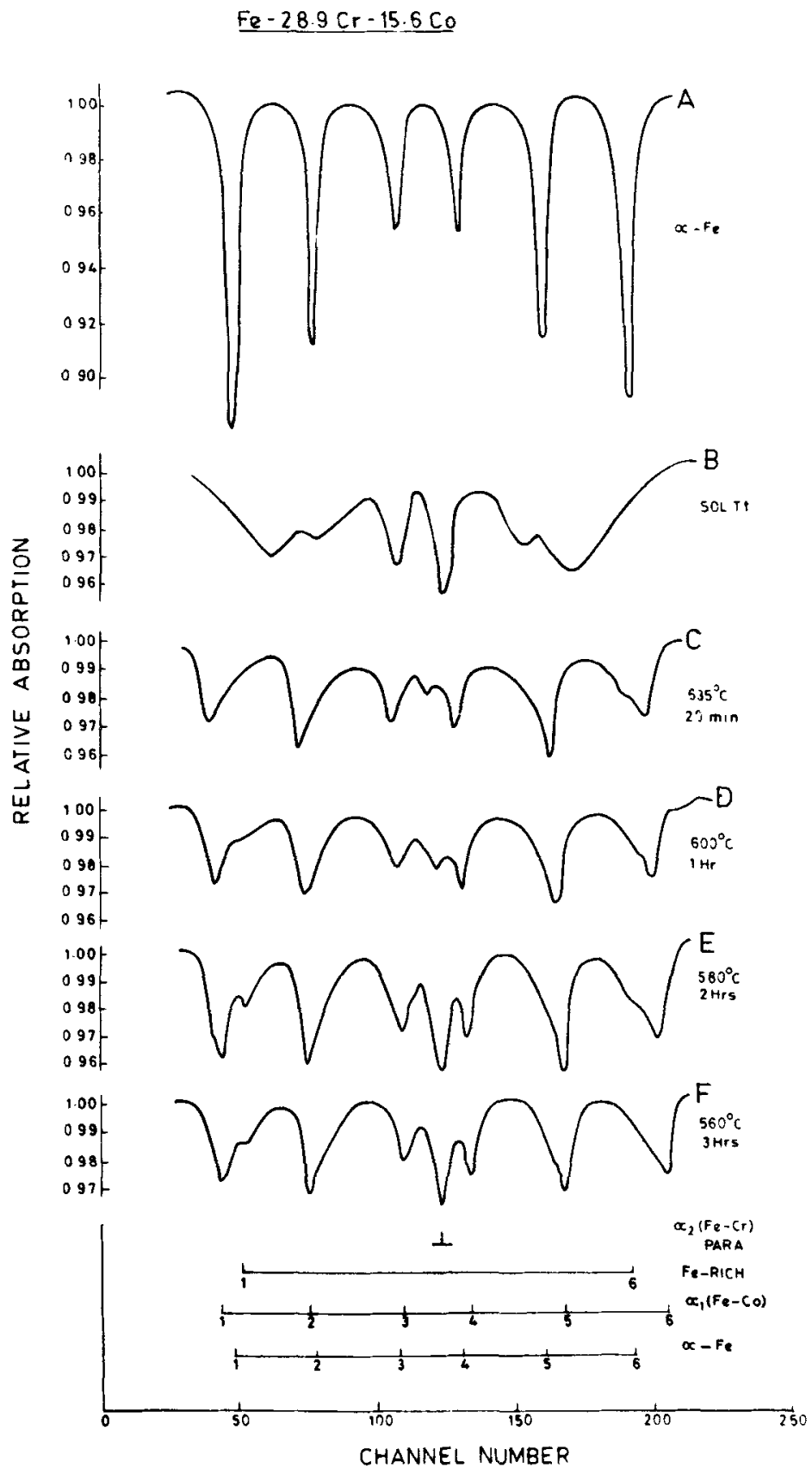

Figure 6. Mössbauer spectra of $\alpha-\mathrm{Fe}$ (for calibration) and alloy 1 at various stages of heat treatment. 
The conversion of weakly ferromagnetic $\alpha_{2}$ to paramagnetic state occurs by the diffusion of Fe-rich phase from $\alpha_{2}$ to $\alpha_{1}$. The migrating Fe-rich phase forms a third intermediate phase whose presence is evidenced by the occurrence of small peaks in the spectrum 6-D to $\mathrm{F}$. The hyperfine field corresponding to this phase has been found to be $320 \mathrm{KOe}$. During the step-tempering treatments, it can be seen that the spectral area of this phase decreases, which may be atrributed to the migration from $\alpha_{2}$ to $\alpha_{1}$. However, no corresponding change in the hyperfine field of $\alpha_{1}$ due to this addition has been noticed.

\section{Conclusions}

The following conclusions are drawn from the present studies.

(i) $\mathrm{Fe}-28.9 \mathrm{Cr}-15.6 \mathrm{Co}$ and $\mathrm{Fe}-28.4 \mathrm{Cr}-20.1 \mathrm{Co}$ alloys develop attractive permanent magnetic properties $B_{r}=11 \cdot 5-12.0 \mathrm{~K}$ Gauss, $H_{c}=600-650 \mathrm{Oe}$ and $(\mathrm{BH})_{\max }$ $=4 \cdot 0-4 \cdot 5 \mathrm{MGOe}$.

(ii) The coercivity of the alloys is sensitive to thermomagnetic treatment and improves gradually upon step-tempering treatment. The remanence of the alloys depends on the strength of the external magnetic field applied during TMT and is not altered during step-tempering.

(iii) TMT parameters such as $635^{\circ} \mathrm{C}, 60$ minutes time and 2000 Oe field are characteristic for alloy 1 while $645^{\circ} \mathrm{C}, 30$ minutes time and 2000 Oe field are so for alloy 2 .

(iv) The widely accepted Stoner and Wohlfarth theory for coercivity in Alnico alloys has been applied to explain qualitatively the experimental observations in $\mathrm{Fe}-\mathrm{Cr}$ Co alloys.

(v) From Mössbauer studies, it has been confirmed that soon after TMT, the alloy develops a strongly ferromagnetic phase $\alpha_{1}$ and a second phase $\alpha_{2}$ existing in weakly ferromagnetic and paramagnetic states. On step-tempering, ferromagnetic $\alpha_{2}$ becomes paramagnetic with the presence of a third intermediate Fe-rich phase.

\section{Acknowledgements}

The authors gratefully acknowledge the help rendered by their colleagues in Chemical Analysis, Technical Assistance, $x$-ray, Optical and Electron Microscopy divisions. Thanks are due to Dr P Rama Rao, Director, DMRL for his keen interest.

\section{References}

Belli Y, Okada M, Thomas G, Homma M and Kaneko H 1978 J. Appl. Phys. 492049

Chen Ming Yan, Wang Hui Juan, Yan Yong, Shao Han Ru, Li De Xin, Lin Wen Gui and Liguo Dong 1982 J. Appl. Phys. $\mathbf{5 3} 2377$

Chin G Y 1978 J. Magn. Mag. Mat. 9283

Chin G Y 1980 Science 208888

Chin G Y, Plewes J T and Wonsiewicz B C 1978 J. Appl. Phys. 492046

Chin T S, Chang C Y, Wu T S, Hsu T K and Chang Y H 1983a IEEE Trans. Mag. MAG-19 2035

Chin T S, Wu T S, Chang C Y, Hsu T K and Chang Y H 1983b J. Mater. Sci. 181681

Cremer R and Pfeiffer I 1975 Physice B80 164

Gin S and Gayle N V 1980 IEEE. Trans. Mag. MAG-16 526 
Green M L, Sherwood R C, Chin G Y, Wernick J H and Bernardini J 1980 IEEE. Trans. Mag. MAG-16 1053 Homma M 1977 Proc. 1st International Seminar on Magnetism, Sebnitz, GDR, 1

Houghton M E, Rossitier P L and Clark P E 1978 J. Mater. Sci. 13155

Kaneko H, Homma M, Fukunaga T and Okada M 1975a IEEE. Trans. Mag. MAG-11 1140

Kaneko H, Homma M and Minowa T 1976 IEEE. Trans. Mag. MAG-12 977

Kaneko H, Homma M and Miura M 1972 IEEE. Trans. Mag. MAG-8 347

Kaneko H, Homma M and Nakamura N 1971 AIP Conf. Proc. 51088

Kaneko H, Homma M, Nakamura K, Okada M and Thomas G 1977 IEEE. Trans. Mag. MAG-13 1325

Kaneko H, Homma M, Okada M, Nakamura S and Ikuta N 1975b AIP. Conf. Proc. 29620

Livingston J D 1981 J. Appl. Phys. 522544

Mahajan S, Gyorgy E M, Sherwood R C, Jin S, Makahara S, Brasen D and Eibschutz M 1978 Appl. Phys. Lett. 32688

Mason J J, Ashall D W and Dean A V 1970 Cobalt 4623

Minowa T, Okada M and Homma M 1980 IEEE. Trans. Mag. MAG-16 529

Okada M, Thomas G, Homma M and Kaneko H 1978 IEEE. Trans. Mag. MAG-14 245

Sherwood R C, Tin S and Chin G Y 1979 IEEE. Trans. Mag. MAG-15 1774

Stoner E C and Wohlfarth E P 1948 Phil. Trans. R. Soc. A240 599

Tin S 1979 IEEE. Trans. Mag. MAG-15 1748

Tin S, Chin G Y and Wonsiewicz B C 1980a IEEE. Trans. Mag. MAG-16 139

Tin S, Mahajan S and Brasen D 1980b Met. Trans. A11 69

Wang Run, Chen Jufang and Zhou Shouzeng 1984 J. Appl. Phys. 552109

Zijlstra H 1978 IEEE. Trans. Mag. MAG-14 661 